\title{
Development of a High-reliability Model for Public Hospitals in Iran
}

\author{
Ghahraman Mahmoudi ${ }^{1}$, Mohammad Ali Jahani ${ }^{2}$, Mousa Yaminfirooz ${ }^{3} \&$ Ramin Navaie $^{4}$ \\ ${ }^{1}$ Department of Health Services, Sari Branch, Islamic Azad University, Sari, Iran \\ ${ }^{2}$ Social Determinants of Health Research Center, Babol University of Medical Sciences, Babol, Iran \\ ${ }^{3}$ Department of General Education, Faculty of Medicine, Babol University of Medical Sciences, Babol, Iran \\ ${ }^{4}$ Master of Science, Health Services Management, Mazandaran University of Medical Sciences, Sari, Iran \\ Correspondence: Ramin Navaie, Mazandaran University of Medical Sciences, Sari, Iran. Tel: 98-911-220-9572. \\ E-mail: raminnavai55@gmail.com
}

Received: October 11, 2016 Accepted: November 7, 2016 Online Published: November 12, 2016

doi:10.5539/gjhs.v9n1p155 URL: http://dx.doi.org/10.5539/gjhs.v9n1p155

\begin{abstract}
Introduction: As a health care organization, achieving security and high reliability is a goal in hospitals. Therefore, this research is concerned with the development of a structural high-reliability model in public hospitals of Iran.
\end{abstract}

Methodology: This applied research was conducted in 2015 with a population that included directors, managers, faculty members and hospital affairs experts. A totalof 200 questionnaires were distributed in five areas of the country based on a cluster sampling method. The structural validity of the questionnaire was approved through confirmatory factor analysis test and its reliability was calculated as 0.73 by Cronbach's alpha test. Moreover, data were analyzed by SPSS 18 and LISREL 8.5 software using factor analysis and mathematical models; and the confirmation of the model was assessed based on confirmatory factor analysis.

Findings: The results of factor analysis indicated that exploitable factors for factor analysis included selection of axis of reluctance to simplify interpretation, preoccupation with failure, sensitivity to operations, resilience and deference to expertise. Conversely, the ratio between Chi-Do index and degree offreedom was less than three, the RMSEA index was lower than 0.08, The NFI, NNFI, IFI and CFI values were higher than 0.9.

Conclusions: By considering the axes of lack of tendency towards simplification of interpretations, concerns and tolerance against failure, sensitivity towards operations, flexibility and specialization hospitals will be able to achieve high-reliability criteria.

Keywords: model, high-reliability, security, hospitals

\section{Introduction}

High reliability organizations are organizations thathave been successful in keeping away from environmental disasters. Such environments are those in which usual errors (as a result of existence of certain factors and risky complications) are likely to occur (Onyiriuba, 2015). In this regard, the structure and management of a health care providing organization can minimize the likelihood of risk occurrence and errors (He et al., 2015). High-reliability organizations are those with risky and complex characteristics that are entirelysecure and effective (Haugan \& Drageset, 2014). Nowadays, it is challenging to definea high-reliability organization as well ascomprehendthe manner of foundation of such an organization. In fact, an organization with high reliability can only attain this position after experiencing several failures (Peng, Zhang, \& Zhu, 2015). These organizations could be identified by providing answers to the question that follows: how often has this organization experiencedfailuresthatcould have resulted indisastrous consequences but were averted? (Andriulo, Arleo, de Carlo, Gnoni, \& Tucci, 2015).

Having a desirable performance is an element thatcan turn a hospital into a high-reliability organization (Conceição, Borges, \& Ascenso, 2015). In other words, high-reliability hospitals should be focused on recognizing errors and their manners of occurrence (Vogus \& Sutcliffe, 2007). According to announced statistics, medical malpractice cases in Iran exceeds 3000, and in approximately $50 \%$ of these cases, physicians are found guilty for different reasons such as carelessness, lack of experienceor sufficient knowledge, and use of inappropriate techniques (Tammelin \& Qvarfordt, 2015). According to published statistics, an American 
healthcare organization has announced that mortalities due to medical malpractice in this country are between 44 to 98 thousand cases per year and in line with these data, medical malpractice is regarded as one of the ten main causes of death in America, taking its place between the fifth and ninth position (Riley, Meredith, \& Parrotta, 2014). In addition to causing harm and inabilities, medical malpractices can prolong the hospitalization period, lead to extraordinary actions and increase medical expenses. Nowadays, as a result, reduction and elimination of such errors as well as increasing the security of health care has become a global priority (Salata, de Lieto Vollaro, de Lieto Vollaro, \& Davoli, 2014).

Despite the importance of analyzing unpleasant events and medical malpractices, it appears that Iran's hospitals still lack a system for identifying and logging errors (Jahani, Naghshineh, Naghavian, \& Semnani, 2013). One of the most important issues regarding the program of dominance of healthcare services is patients' security (Thomassen et al., 2011). In addition, managers and experts in the system are looking for a way to provide more secure and reliable healthcare (Wright, 2015). In fact, in terms of performance and continuous processing, it is highly useful and beneficial to regard health care providing organizations as institutions required to structuralize high-reliability systems and institutionalize the system - a continuous activity that should be followed up (Vogus \& Sutcliffe, 2007). High-reliability healthcare calls for standardization of medical methods which is also a requirement for the patient's security (Kavari, 2006). Also, provision of job satisfaction as well as consideration of staff status is a primary concern (Niedner, Muething, \& Sutcliffe, 2013). In addition, these stations provide various services and different offices are assigned to legislate and control them. Consequently, coordinating programs that are collectively provided in a hospital, and have each been started from a different route seems simultaneously necessary and challenging (Steel, 2015). On this basis, as great national capitals, hospitals have many different equipment and facilities (Winston, Sallis, Swartz, Hoelscher, \& Peskin, 2013).

Sujan (2015) showed that health and treatment organizations support a better understanding of practical processes of organizational learning in a national context (Sujan, 2015). The criteria of free flow of information, continuous optimization, reporting errors, collaboration of patients' families, leadership of organizational security, and leadership of professional security and access to equipment and rules have been used for development of a scale for patient's security atmosphere in Japan (Matsubara, Hagihara, \& Nobutomo, 2008). Achieving high-reliability was feasible through supportive leadership and conceptual model in goals' contact establishment, utilization of effective optimization methods and ensuring responsiveness in John Hopkins Hospital (Pronovost et al., 2013). High-reliability organizationmaintain sits stability for a long period of time. In all aspects of health and treatment, the status of a high-reliability organization is viewedas the next step towards optimization of security and quality (May, 2013). Based on this, the present research attempted to develop a high-reliability model for public hospitals by studying the factors that affect high-reliability.

\section{Materials and Methods}

This research is an applied study conducted in 2015 and prepared through modeling for structural equations. Its population comprises C.E.Os, managers and hospital experts which include physicians, faculty members of Universities, master specialists and specialists of hospital fields. According to K.M.O, if the p-value islessthan 0.05 in structural equations modeling, it is an indicationthat the sample size is sufficient. According to this method, 200 individuals were selected as the sample among the aforementioned population (Fox, 2006). The sampling was done through a cluster method with the entire country divided into five segments (north, south, center, east and west), two hospitals in each segment were selected and 20 quotas were assigned to each hospital. The questionnaires were filled out by participants in a stratified random fashion. The terms for entering the study were conscious agreement of the participants who volunteered for the study.

By studying books and various articles in different languages and by taking help from valid scientific information and the internet, the key models of high-reliability were theoretically stated and further analyzed. Based on data obtained from the prior and post steps to exploitation of the main dimensions of the model and a consideration ofthe hospital's special characteristics as well as other environmental conditions, a questionnaire was developed. To determine content validity, the questionnaire was forwarded to twenty professors and faculty members of different universities outside the research population; of these, fifteen studied the questionnaire and returned it. By employing their suggestions and ideas, necessary reformations were made on the questions; thereafter, the final questionnaire was developed and distributed among the sample. To determine the reliability of the questionnaire used in this research, about 30 questionnaires were distributed among the sample and after collection, the Cronbach's alpha coefficient of the questionnaire was calculated as 0.73 . The questionnaire comprised two sections: the first section included demographic features in five questions whilethe second section contained the main questions of the survey regarding the high-reliability of public hospitals in 54 closed questions and 6 items. The scale used in this questionnaire was the 5-degrees Likert scale. Due to the possible 
loss of 250 questionnaires were distributed among the sample and of this number, 200 were selected as analyzable, and were collected back. Factor analysis was performed on the data which resulted from the questionnaires and the final model was developed by utilizing mathematical models. Furthermore, the model was confirmed using confirmatory factor analysis. Both SPSS v18.0 and LISREL v8.50 software were employed for data analysis.

\section{Findings}

According to the findings of this research, 55\% of the sample was females, $29 \%$ were between $37-42$ years old, $51 \%$ held an M.A degree, $32.5 \%$ of them had experience of between $13-18$ years while $36.9 \%$ had experience of between 2-9 years. It is noteworthy to state that most of the participants had studied healthcare services management (Table 1).

Table 1. Abundance distribution, abundance percentage and cumulative percentage of demographic variables of respondents

\begin{tabular}{|c|c|c|c|c|c|}
\hline Variables & & Abundance & $\begin{array}{l}\text { Observed } \\
\text { abundance }\end{array}$ & $\begin{array}{l}\text { Observed } \\
\text { percentage }\end{array}$ & $\begin{array}{l}\text { Cumulative } \\
\text { percentage }\end{array}$ \\
\hline Gender & & Female & 110 & 55 & 100 \\
\hline Age & & $37-42$ & 58 & 29 & 73 \\
\hline Education & & M.A & 102 & 51 & 86.5 \\
\hline Experience & & $13-18$ years & 65 & 32.5 & 78 \\
\hline $\begin{array}{l}\text { Field } \\
\text { education }\end{array}$ & of & $\begin{array}{l}\text { Healthcare services } \\
\text { management }\end{array}$ & 94 & 47 & 88 \\
\hline
\end{tabular}

With respect to sufficiency of K.M.O value and Bartlett's sphere city significance test, the data are suitable for factor analysis (Table 2).

Table 2. K.M.O value and results of Bartlett's sphere city significance test for the high-reliability of Iran's public hospitals

\begin{tabular}{lll}
\hline Index & Suitable value & Calculated value \\
\hline K.M.O & Higher than 0.7 & 0.785 \\
Bartlett's significance & Lower than 0.05 & 0.000 \\
Test result & & Suitable \\
\hline
\end{tabular}

From the output of LISREL software (Figure 1), the calculated values of fitness index are suitable and the entire load factors are higher than 0.3 . 


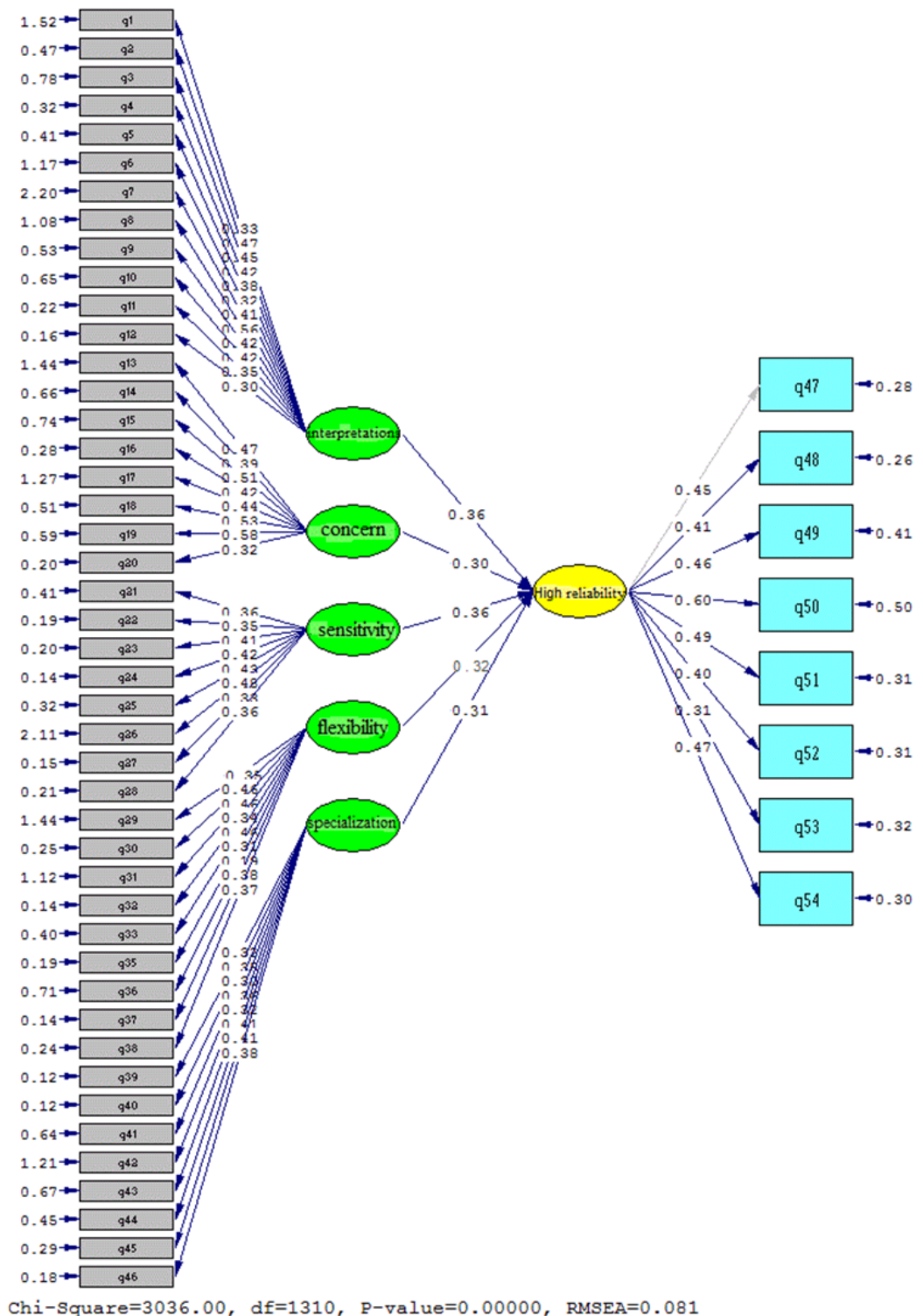

Figure1. Standard load factors of structural model of research for effective elements on high-reliability of Iran's public hospitals

The results displayed above indicate that in terms of indexes of fitness, the research model is in a suitable situation. Also for the root average error of approximate squares with the acceptability range of smaller than 0.5 , the value of 0.081 is very good. As a result with respect to entire indexes and with respect to calculated fitness indexes it can be said that indexes of research model's fitness are suitable (Table 3).

Table 3. Fitness indexes for research models in terms of effective elements on high-reliability of Iran's public hospitals

\begin{tabular}{lllllllll}
\hline Fitness index & $\chi^{2 / \mathrm{df}}$ & SRMR & RMSEA & GFI & AGFI & NFI & CFI & IFI \\
\hline Fitness for research model & 2.31 & 0.04 & 0.081 & 0.93 & 0.90 & 0.95 & 0.94 & 0.84 \\
Acceptability range & $1-5$ & $<0.05$ & $<0.5$ & $>0.9$ & $>0.9$ & $>0.9$ & $>0.9$ & $0-1$ \\
\hline
\end{tabular}

With respect to thet-testresults, this model's significance of observed correlations is higher than 1.96; hence, all correlations are accepted (Figure 2). 


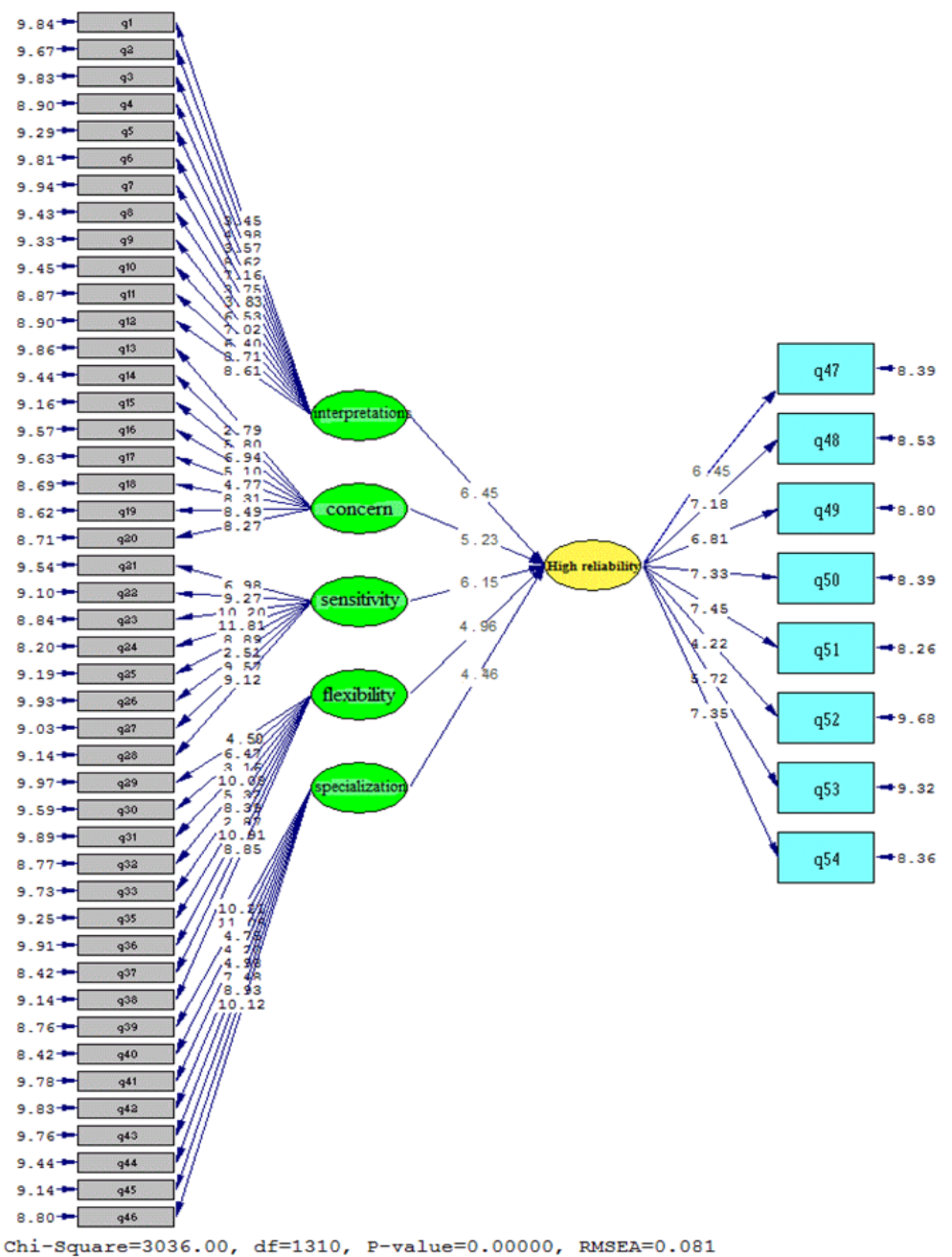

Figure 2. Load factors of the t-value statistic of structural model for effective factors on high-reliability of Iran's public hospitals

\section{Discussion}

Results revealed that regardingthe value of K.M.O index, the sample size was sufficient for performance of factor analysis. In addition, the significance level observed in Bartlett's test indicated the suitability of the factor analysis for recognizing the structure of the factorial model. As regards diagrams related to structural equations model and levels of correlations, all the relations including effective factors on high-reliability as well as high-reliability model were accepted. Also, the output of LISREL software, revealed that the calculated values of fitness index are suitable. On this basis, questions and dimensions related to independent variables (reluctance to simplify interpretation, preoccupation with failure, sensitivity to operations, resilience and deference to expertise) are effective on the high-reliability of hospitals.

In the view of Walston et al. (Walston, Al-Omar, \& Al-Mutari, 2010), the efficiency of organizing high-reliability organizations in the context of medicine is still premature, however evidence have shown that special attention is being redirected towards the paradigm of high-reliability organizations. Hellings et al. (Hellings, Schrooten, Klazinga, \& Vleugels, 2010) stated that the average score of patients' security level in hospital was atan average level and also, there were no significant relationship between direct and indirect interactions with patients, regarding their security level. According to Knox (Knox, Simpson, \& Townsend, 2003), it is necessary to have an effective system of learning from incidents for continuous optimization of capabilities of disaster management processes.

Chassin et al. (Chassin \& Loeb, 2013), elaborated on five main high-reliability factors. Their results revealed that hospitals and other organizations providing health care behave in such a way that failure in routine activities 
is considered inevitable and one of the most prevalent problems in hospitals, as they have stressed out their sensitivity to operations. Roger (Rogers \& Wright, 1998) has described the symptoms of a high-reliability organization in terms of other industries with these symptoms including concepts such as group awareness and consideration, concerns and tolerance against failure. However, the former researcher did not clearly state this issue but used them in his work. So it can be observed that in these high-reliability non-healthcare organizations, common processes are more trusted than processes which exist in healthcare providing organizations. Concerns and tolerance against failure is an important basis for providing high-reliability organizations with a different kind of quality. For high-reliability organizations, concerns are accompanied with something that is perceived seldom. Although high-reliability organizations successfully keep avoiding incidents, they do not exaggerate their advantage. In addition, high-reliability organizations aremore cautious regarding common failures after victory. These failures are mostly due to limited searching, insufficient consideration as well as incompatibility of risks (Rogers \& Wright, 1998). Additionally, May et al, took cognizance of the staff awareness factor and has therefore considered awareness as one of the five elements stated by Vick and Satalif (May, 2013). As a combined series of features, these characteristics provide trust worthy processes with the least amount of possible errors. Organizations providing healthcare have shown their interest in high-reliability but have not clearly established the steps for achieving it. As soon as the patient's security knowledge gets deeper, healthcare towards awareness and a more efficient high-reliability becomes clearer (May, 2013).

There is a relation between multi-sectorial dimensions of Iran's public hospitals; hence, if public hospitals areto be regardedas high-reliability organizations, then they should emphasize more on increasing quality as well as reinforcement of knowledge and staff learning. Hospitals should also be responsible for possible incidents. In doing so, they are expected to pay attention to the following five main features. These features include strength for tolerance of failure in programs, lack of tendency for simplification of analyses and interpretations, being sensitive towards desirable performance, flexibility against problems and specialization. The effects of these high-reliability factors are not equal in Iran's public hospitals as observed in the results of this research. Also, Chassin(Chassin\& Loeb, 2013), studied the five main factors of high-reliability organizations and proposed ideas that are in contrast with the requirement for becoming a high-reliability organization. In terms of their view, there were no correlation and relations between multi-sectorial dimensions of hospitals. Roger et al., investigated the symptoms of a high-reliability organization in relation to other industries. These symptoms included group awareness as well as concerns and tolerance against failure. However, the type of relation between these factors was not described. Rather, it was only stated that these factors had a higher and greater correlation in non-healthcare organizations (Rogers \& Wright, 1998). To some extent, these results are consistent with the obtained results of the present research in which the entire relations were approved. Furthermore, in the present research, the entire effective dimensions on high-reliability of hospitals were simultaneously investigated and a related model was there after developed.

\section{Conclusion}

By considering the following factors: reluctance to simplify interpretation, preoccupation with failure, sensitivity to operations, resilience and deference to expertise hospitals need to obtain the criteria for qualifying as a high-reliability organization.To achieve the status of a high-reliability hospital, first, the existing defects in the hospital should be realized and taken care of. Also, the status of high-reliability dominancein the station for managers needs to be clarified and subsequently help to improve its status by increasing employees' awareness about patients' security. After making necessary interventions for improvement of security, managers can use it as an instrument for evaluating these interventions. Iran's public hospitals should act as flexible organizations and should also be able to assign their entire resources to the entire medical needs of the society. This coordination however, requires the knowledge and art of the highest level of hospital management. By using several creative transformations of different organizations, hospitals can make important progressions towards becoming a high-reliability organization. Finally, it should be stated that making progress towards formation of these aforementioned factors requires a secure culture.

Every research is bound to certain limitations and among the limitations of this research, there is lack of tendency among some of the hospital personnel as well as lack of previously conducted researches in a subject similar to this research.

\section{Acknowledgments}

The authors appreciate the cooperativeness of authorities, the entire hospitals considered through out the country and their sincere experts and specialists. 


\section{Competing Interests Statement}

The authors declare that there is no conflict of interests regarding the publication of this paper.

\section{References}

Andriulo, S., Arleo, M. A., de Carlo, F., Gnoni, M. G., \&Tucci, M. (2015). Effectiveness of maintenance approaches for High Reliability Organizations. IFAC-PapersOnLine, 48(3), 466-471.https://doi.org/10.1016/j.ifacol.2015.06.125

Chassin, M. R., \& Loeb, J. M. (2013). High - reliability health care: Getting there from here. Milbank Quarterly, 91(3), 459-490. https://doi.org/10.1111/1468-0009.12023

Conceição, C., Borges, J., \&Ascenso, R. M. T. (2015).The Influence of Information Systems in the Management of Patients Service in the Hospital of Leiria.Procedia Computer Science, 64, 40-46. https://doi.org/10.1016/j.procs.2015.08.461

Fox, J. (2006). Teacher's corner: structural equation modeling with the sem package in R. Structural equation modeling, 13(3), 465-486. https://doi.org/10.1080/10705511.2012.713275

Haugan, G., \&Drageset, J. (2014).The Hospital Anxiety and Depression Scale-Dimensionality, reliability and construct validity among cognitively intact nursing home patients.Journal of affective disorders, 165, 8-15. https://doi.org/10.1016/j.jad.2014.04.042

He, Z., Liu, R., Wu, P., Zhai, J., Lai, T., Song, S., \& Song, Z. (2015). High speed and high reliability in Ge 8 Sb 92/Ga $30 \mathrm{Sb} 70$ stacked thin films for phase change memory applications. Journal of Alloys and Compounds, 653, 334-337. https://doi.org/10.1016/j.jallcom.2015.09.008

Hellings, J., Schrooten, W., Klazinga, N. S., \&Vleugels, A. (2010). Improving patient safety culture.International Journal of Health Care Quality Assurance, 23(5), 489-506. https://doi.org/10.1108/09526861011050529

Jahani, M. A., Naghshineh, A., Naghavian, M., \&Semnani, H. (2013).Safty indicators in the hospitals affiliated to Baboluniversity of medical Sciences,Iran,2010,15(2),95-101.

Kavari, S. H. (2006). A study of depression prevalence in nurses and it's effect in Shiraz Namazi hospital. Middle East J Fam Med, 4(3), 17-21. https://doi.org/10.1016/S0924-9338(11)72346-X

Knox, G. E., Simpson, K. R., \& Townsend, K. E. (2003). High reliability perinatal units: further observations and a suggested plan for action. Journal of Healthcare Risk Management, 23(4), 17-21. https://doi.org/10.1002/jhrm.5600230405

Matsubara, S., Hagihara, A., \&Nobutomo, K. (2008).Development of a patient safety climate scale in Japan.International Journal for Quality in Health Care, 20(3), 211-220. https://doi.org/10.1093/intqhe/mzn003

May, E. L. (2013). The power of zero: steps toward high reliability healthcare. Healthcare executive, 28(2), 16-8,20,22passim.

Niedner, M. F., Muething, S. E., \& Sutcliffe, K. M. (2013).The high-reliability pediatric intensive care unit.Pediatric Clinics of North America, 60(3), 563-580. https://doi.org/10.1016/j.pcl.2013.02.005

Onyiriuba, L. (2015). Emerging Market Bank Lending and Credit Risk Control: Evolving Strategies to Mitigate Credit Risk, Optimize Lending Portfolios, and Check Delinquent Loans: Academic Press.

Peng, W., Zhang, J., \& Zhu, D. (2015).ABCLS method for high-reliability aerospace mechanism with truncated random uncertainties.Chinese Journal of Aeronautics, 28(4), 1066-1075. https://doi.org/10.1016/j.cja.2015.06.012

Pronovost, P. J., Demski, R., Callender, T., Winner, L., Miller, M. R., Austin, J. M., \&Berenholtz, S. M. (2013). Demonstrating high reliability on accountability measures at the Johns Hopkins Hospital. The Joint Commission Journal Quality and Patient Safety, 531-544.https://doi.org/10.1016/S1553-7250(13)39069-2

Riley, W. J., Meredith, L., \&Parrotta, C. (2014).Measuring High Reliability in Hospital Perinatal Units.GSTF Journal of BioSciences (JBio), 2(1),104-109. https://doi.org/10.5176/2251-3140_2.1.30

Rogers, E. W., \& Wright, P. M. (1998).Measuring organizational performance in strategic human resource management: Problems, prospects and performance information markets.Human resource management review, 8(3), 311-331. https://doi.org/10.1016/S1053-4822(98)90007-9 
Salata, F., de LietoVollaro, A., de LietoVollaro, R., \&Davoli, M. (2014).Plant reliability in hospital facilities.Energy Procedia, 45, 1195-1204. https://doi.org/10.1016/j.egypro.2014.01.125

Steel, C. (2015).Auditing Clinical Practice: Ensuring High Reliability at an Australian Hospital.AORN journal, 102(1), 81-84. https://doi.org/10.1016/j.aorn.2015.05.013

Sujan, M. (2015). An organisation without a memory: A qualitative study of hospital staff perceptions on reporting and organisational learning for patient safety. Reliability engineering \& system safety, 144, 45-52. https://doi.org/10.1016/j.ress.2015.07.011

Tammelin, A., \&Qvarfordt, I. (2015).Point-prevalence surveillance of healthcare-associated infections in Swedish hospitals, 2008-2014.Description of the method and reliability of results.Journal of Hospital Infection, 91(3), 220-224. https://doi.org/10.1016/j.jhin.2015.07.013

Thomassen, O., Espeland, A., Softeland, E., Lossius, H. M., Heltne, J. K., \&Brattebo, G. (2011). Implementation of checklists in health care; learning from high-reliability organisations. Scand J Trauma ResuscEmerg Med, 19(53), 1-7. https://doi.org/10.1186/1757-7241-19-53

Vogus, T. J., \& Sutcliffe, K. M. (2007). The safety organizing scale: development and validation of a behavioral measure of safety culture in hospital nursing units. Medical care, 45(1), 46-54. https://doi.org/10.1097/01.mlr.0000244635.61178.7a

Walston, S. L., Al-Omar, B. A., \& Al-Mutari, F. A. (2010). Factors affecting the climate of hospital patient safety: A study of hospitals in Saudi Arabia. International Journal of Health Care Quality Assurance, 23(1), 35-50. https://doi.org/10.1108/09526861011010668

Winston, C. P., Sallis, J. F., Swartz, M. D., Hoelscher, D. M., \&Peskin, M. F. (2013). Reliability of the hospital nutrition environment scan for cafeterias, vending machines, and gift shops. Journal of the Academy of Nutrition and Dietetics, 113(8), 1069-1075. https://doi.org/10.1016/j.jand.2013.04.004

Wright, S. M. (2015). Patient Safety in Anesthesia: Learning from the Culture of High-Reliability Organizations. Critical care nursing clinics of North America, 27(1), 1-16.https://doi.org/10.1016/j.cnc.2014.10.010

\section{Copyrights}

Copyright for this article is retained by the author(s), with first publication rights granted to the journal.

This is an open-access article distributed under the terms and conditions of the Creative Commons Attribution license (http://creativecommons.org/licenses/by/4.0/). 